\title{
TOWARDS A DUAL ONTOLOGY: DUALITY, A CASE STUDY
}

\author{
PARA UMA ONTOLOGIA DUAL: DUALIDADE, UM ESTUDO DE CASO
}

FRANCESCO MARIA FERRARI ${ }^{1}$

Universidade Estadual de Campinas (UNICAMP) - Brasil

ferrarifram@gmail.com

\begin{abstract}
The main aim of this work is to depict the relevance of one of the most interesting formal concepts, i.e., duality, with respect to some foundational issues related to the actual ontological debates arising from the epistemological analysis of physical processes. In particular, it is argued that a formal characterization of emergence is possible in terms of the category theoretic notion of duality. This would constitute a significative shifting from a Platonic to an Aristotelian ontology (of mathematics and, then, of physics). Actually, providing a contribution to this very shift is what this paper wants to be focused on.
\end{abstract}

KEYWORDS: Category theory. Duality. Dynamic system. Dual ontology. Emergence. Modal logic.

RESUMO: O principal objetivo deste trabalho é apresentar a relevância de um dos mais interessantes conceitos formais, i.e., dualidade, concernente a algumas questões fundamentais relacionadas aos debates ontológicos atuais que surgem de uma análise epistemológica dos processos físicos. Em particular, se argumenta que uma caraterização formal da emergência é possível em termos de dualidade em teorias das categorias. Isto constitui uma mudança significativa da ontologia platônica para uma aristotélica (da matemática e, portanto, da física). De fato, fornecer uma contribuição a essa mudança é o que este artigo pretende se concentrar.

PALAVRAS-CHAVE: Teoria das categorias. Dualidade. Sistema dinâmico. Ontologia dual. Emergência. Lógica modal.

\section{INTRODUCTION}

The main aim of this work is to depict the involvement of the concept of emergence by duality - such a concept is at the borderline between modal logic $(\mathrm{ML})^{2}$ category theory $(\mathrm{CT})^{3}$ - in some epistemological issues relative to

\footnotetext{
${ }^{1}$ Pesquisador associado à Universidade Estadual de Campinas - Centro de Lógica, Epistemologia e História da Ciência (UNICAMP - CLE).

${ }^{2}$ Modal logic is the logic of necessity and possibility (operators) and their intensional interpretations, such as, obligation and permission (deontic logic); past and future (temporal logic), etc.

${ }^{3}$ Category theory formalizes mathematical structure and its concepts in terms of a labeled directed graphs called a category, whose nodes are called objects, and whose labelled directed edges are called arrows (or morphism), basically, relations. A category has two basic properties: the ability to compose the arrows associatively and the existence of an identity arrow for each object. The
} 
fundamental physics and to draw some conclusions about the ontology behind such frameworks.

Current fundamental physics is based on the dynamic approach to the quantum field, whose range is made of non-isolated systems and whose basic mechanism of symmetries (patterns) formation supposes the spontaneous breaking of symmetries (SBS). SBS implies that such systems reach unpredictable states and that the information displayed by a system at a fixed state is always incomplete with respect to its evolution (BAILLY; LONGO, 2013). A question, thus, arises about the ontology of such systems, for an ontology of objects seems to be very implausible. The point, it seems, is that the most widespread formalized ontologies supposes the actual existence of objects, by conceiving basic entities just as objects. ${ }^{4}$ On the contrary, it seems, evolutive and non-isolated (open) systems are to be conceived primarily as processes and just in a secondary "emergent" way as objects.

Being a process, in opposition to being an object, means being an incomplete entity with respect to the information displayed at a certain state (or moment) of its evolution (ROVELLI 2016). What makes a process informationally complete and, thus, an actually existent entity (i.e., an object) is the stability it can reach in non-isolation conditions, that is, through the continuous and mutual matching with the energy and information provided by the external (as well as internal) environment. In this sense, the process is stable or is an object until the pair system-environment plays a dual game.

The issue where the formal import of ML and of CT converge is precisely the contingent (modal) existence of objects, things as stable processes. Further, such a stability, such a contingent existence proper of things is governed by the duality between the physical system and its environment.

Evolutive systems, from thermodynamics to evolutive biology, are characterized by irreversibility of states. But since natural science abandoned ontology, asymmetries such as cause-effect relation or processes have been abandoned in favour of algebraic laws, equations. But the language of algebra is symmetric: if $\mathrm{x}$ tells us about $\mathrm{y}$, then $\mathrm{y}$ tells us about $\mathrm{x}$ - and physics became grounded on deterministic relationships. There is no way to write in mathematics a simple irreversible facts - for example, that the upcoming storm causes the barometer to go down, and not the other way around. Mathematics has not developed the asymmetric language required to capture asymmetrical laws describing irreversible phenomena, f.i., that x causes $y$, which does not mean that y causes $x$. It seem that we lack a calculus for asymmetrical relations.

language of category theory has been used to formalize concepts of other high-level abstractions such as sets, ring and groups. Categories are algebraic structures with many complementary natures, e.g., geometric, logical, computational, combinatorial.

${ }^{4}$ Think of the most commonly used formal semantics, i.e., set theoretic semantics. In general, nonactualist ontologies differs only for they employ partial (denoting and/or interpretation) functions. This allow them to speak of non-existent objects inasmuch not all terms denotes. The logical context that derives is that of free logics, i.e., free of existential presuppositions. 
It turned out that the notion of duality, as it is conceived formal sciences in particular in CT - may play a relevant role here. If algebra was discovered since ancient times and, later, applied to the physics of isolate systems (viz., Newtonian mechanics), another category of mathematical tool has been discovered within $\mathrm{CT}^{5}$ in very recent times: that of coalgebra. And coalgebra(s) is(are) dual to algebra(s). To come back to the duality system-environment, such a duality seems to be well captured by mathematics through the duality between algebra and coalgebra. Perhaps, the link between the pairs system-environment and algebra-coalgebra seem to be not just a matter of chance, of subjective choice.

\section{ACTUALISM VS DUAL ONTOLOGY}

It is claimed that the fundamental difference and advantage of CT approach to foundation with respect of the set theoretic one is that, instead of considering objects and operations for what they "are" - as it is in set theory and set theoretic logic and ontology - it considers them for what they "do" (ABRAMSKY, 2012). This, of course, would constitute a significative shifting in mathematical philosophy: precisely, from a Platonic to an Aristotelian ontology (of mathematics and of physics). Actually, this very shift is what this paper want to be focused on. In fact, the implicit point this investigation is concerned with is how to treat potentiality.

The modalisation of the existence is the very point at work. Actuality, here as elsewhere, contrasts with potentiality. But the Aristotelian notion of potentiality differs from all the most widespread ontologies such as Platonism, ${ }^{6}$ conceptualism and nominalism ${ }^{7}$ inasmuch it does not presuppose any actuality. ${ }^{8}$ In fact, it is well known that the Platonic presupposition of set theory consists in the fact "that each potential infinite, if it is rigorously applicable mathematically, presupposes an actual infinite" (HALLETT, 1984, p. 25).

Certainly, a real modalisation of ontology cannot be committed to any presupposition of actuality, for in this case potentiality would be reduced to actuality itself. Indeed, any presupposition of actuality means a static ontology. Gill (2003), correctly, reminds us that Aristotle's conception of being is dynamic, that he complained that the source of becoming as irreversible change for atomistic collisions remained unexplained. Aristotle, indeed developed a philosophy of

\footnotetext{
${ }^{5}$ Eilenberg and Mac Lane (1945) introduced categories in a purely auxiliary fashion, as preparation for what they called "functors" and "natural transformations".

${ }^{6} \mathrm{I}$ am distinguishing within realism between (at least) Platonism and natural realism. Atomism, in mathematical contexts, is quite uninteresting for it is formally equivalent to nominalism.

${ }^{7}$ See, for a precise and formal account of such ontologies (COCCHIARELLA, 2007, 2001).

${ }^{8}$ See (GARSON, 2001) for what concerns the objectual and intensional (conceptual) interpretation of quantified ML (QML). In particular, the main reason for the conceptual interpretation of physical reality is that "things, since they change, cannot be identified with term extension. Instead, [...] they correspond to term intensions or individual concepts" (p. 281). But, there are a lot of problem with this. The main, Garson stresses, is that given that individual concepts are functions from indices or possible worlds to objects, quantifying over them means that "we interpret the domain of any quantifier as a set of all functions" and, then, "we run the risk that the language will have the expressive power of second-order arithmetic, with the result that Gödel's Theorems applies" (p. 282).
} 
nature - his hylemorphism - that speaks of natural entities. Such entities contingently persist by (a) the active internal process organization (morphe, or 'form') that realizes a characteristic sort of behaviours and by means of which entities are sorted into natural kinds and (b) the passive internal process, a matter or energy (hylé) that supports the relevant process organizations but that also harbors counteracting. Against any dualism, form and matter are not substances or per sé existing entities (id quod, for the latin tradition) but, instead, constitute each substance as dual correlate processes (id quo) - linked to act and potency respectively. In this sense Aristotelian ontology is not dualistic but, specifically, dual.

\section{FOUNDATIONAL AND EPISTEMOLOGICAL PREMISES}

A preliminary step of this paper consists in tracing a brief discussion on foundation of science and ontology, with special attention to some epistemological and ontological issues belonging to the current physical picture.

\subsection{FOUNDAIIONS AND THE FAILURE OF SCIENIIFIC REALISM}

The endorsement of the belief in the reality of some sort of universal entities ${ }^{9}$ with respect to our best scientific theories, in particular, Scientific Realism (SR), are articulated in two main dimensions:

- an ontic dimension, committed to the mind-independent existence of the world investigated by the sciences and

- a semantic dimension for which claims about scientific primitive entities ${ }^{10}$ should be construed literally as having truth values.

From these a third, epistemic, dimension arises: that those scientific claims constitutes our knowledge of the (external) world.

After the formalization of ontologies (see COCCHIARELLA, 2007) the main trouble for realists is to argue in favour of the semantic and epistemic dimensions. The debates and discussions are, indeed, mostly affected by the belief that the foundamental basic entities must, by logic, be "objects", in any interpretation one wants to give to them. Namely, "they must be individuals or discrete; they must perdure; they must not merge (two objects becoming one); they must not multiply spontaneously (one object becoming two)". But, whatever one may decide for, all these are "substantive metaphysical assumptions" (DUTILH-NOVAES, 2014, pp. 8586) which constitutes an ontological bias - till the scientific method ${ }^{11}$ does not provide a foundation, a grounding, a selection over the plausible choices.

\footnotetext{
${ }^{9}$ I.e., properties, classes, natural kinds, etc.

${ }^{10}$ I.e., events or processes or properties and relations.

${ }^{11}$ I.e., empirical.
} 
Given the (supposed) mathematical nature of reality, ${ }^{12}$ the semantic trouble reduces to the truth of the axioms of set theory, as it is the most widely accepted mathematical theory of objects able to reduce all mathematics, after the aritmetisation of analysis and function theory due to Weiestrass (FRAENKEL; BARHILLEL, 1973). The trouble, indeed, is even epistemic and concerns the formulation of a criterion of $\operatorname{truth}^{13}$ (see CELLUCCI, 2014).

It is widely held that the most powerful argument in favour of scientific realism is the no-miracles argument, according to which the success of science would be miraculous if scientific theories were not (at least approximately) true descriptions of the world. But according to axiomatic method, that the success of scientific theories is not a miracle, i.e., that scientific theories are true, has to be proved in the theory itself. Thus, such a miracle, actually, takes the form of the correspondence between the theory and the external reality. Indeed, scientific realists, who assume truth as possession of a model, are in general also bound to assume that formal structures or theories are isomorphic (homomorphic up to infinite domains) to the external world. But, following Cellucci (2014), "this must be demonstrated in some mathematical theory, and ultimately in Zermelo-Fraenkel set theory (ZF), and presupposes that the axioms of ZF be true. But, by Gödel's Second Incompleteness Theorem, it is impossible to demonstrate by any absolutely reliable means that the axioms of $\mathrm{ZF}$ are true".

By the very same reason, the absolute (independent) existence of formal entities is not demonstrable within the axiomatic method. In fact, from Gödel's Theorems, we know that the formalist approach (Hilbert) to foundations fails together with the Platonic one (Frege) - and, even, the intuitionistc one (Brower). Indeed, we may argue that:

H1. The formalist principle 'consistency (coherence) implies truth (satisfaction)' fails: the truth of ZF system is not provable for it is not provable the absolute consistency of set theory - absolute: in the same mathematical system and by finitary (denumerable or effective) procedures;

H2 The logicist principle 'truth implies existence', from H1 and transitivity of formal implication, fails: even the absolute (independent) existence of sets is not provable.

\footnotetext{
12 This supposition induced the modern "Copernican revolution" in philosophy and metaphysics, started by Descartes and ended by Kant and passed through the Galilean-Newtonian revolution in natural sciences. "Induced", since the foundation of (mathematical) information does not actually need an (transcendental) subject. For three reasons: (i) necessity of mathematics is no longer synthetic, after Quine's Two Dogmas, (ii) nor analytic (if any), after Frege and Russell's paradox, (iii) nor a priori, after Gödel. Information, and hence, mathematical information included, does not presupposes any notion of mind or of (transcendental) subject (see even a very interesting interview of S. Wolfram to Scientific American (HORGAN, 2017).

${ }^{13}$ A criterion of truth differs from the definition of truth for, while the definition allows us to know under which conditions, if satisfied, a statement is true, it allows to know when a statement is true, namely when those conditions are (or not) satisfied. Thus, the criterion is able to select and fix the model that is used to satisfy such conditions.
} 
C The axiomatic (deductive) view of proving the SR stance had failed.

Thus, given that correspondence to the external world and absolute existence of formal objects is not formally provable and since the belief in the truth of our best theories is not miracolous, on what might be axiomately grounded the belief in our best scientific theories?

\subsection{BEYOND REDUCTIONISM AND DETERMINISM IN NATURAL SCIENCE}

The axiomatic approach to foundation has an analogous counterpart in treating the information in physical evolutive systems: the modern paradigm, i.e., the view that started from the very beginning of the Galilean revolution. According to this view, natural processes are linear or, equivalently, natural events are governed by linear causality. ${ }^{14}$ Modern natural science supported such a conception for the only equations we were able to treat at that time were the linear ones. As a consequence, according to the modern paradigm, laws of nature are always representable by linear equations and, thus, integrable. Yet, this formal approach reacted a matter of physical content. As an immediate consequence, linear equations would have formally represented the modern concept of causality, i.e., material and efficient causality. ${ }^{15}$ Accordingly, a system and/or its evolution depend exclusively (and completely) on its initial conditions, under the dependence of what (BRIDGMAN, 1958, p. 174-175) is called the "analysis of a situation into simple [reads: 'mutually independent'] elements". The latter being a condition of isolation and compositionality of any system.

Then is possible to notice a parallel between formal systems [in the mathematical sense (CELLUCCI, 2013)] and modern approach to physical systems that lies on the following analogy (A):

(A) All the information that describes a modern physical and/or formal system is just implicit in the initial conditions and/or in the axioms that describe such a system.

Looking at physical systems in general, this approach embodies two forms of physical reductionism, i.e., determinism:

(i) by reducing the behaviour of complex systems to the linear laws governing the behavior of their elementary constituents (diachronic);

\footnotetext{
${ }^{14}$ Linearity is a property of order, i.e., linear orders. Namely, an order is linear when all the member of a given set may be ordered by the given order; or, equivalently, when it is total (satisfies trichotomy) and transitive (ENDERTON, 1977). Where totality as the specific property of linearity means exactly that any/all elements of the given set are in this order. Notice that linear orders are irreflexive, i.e., it is not the case that any object is in such a given order with itself. Partial orders are in opposition with linear ones, for they are reflexive and not total, but transitive and antisymmetric. These notions will be useful later on in Sect. 4.

${ }^{15}$ Material and efficient causality (initial conditions). Forms (essences), as well as final causes (terminal conditions) - whatever intended - were intentionally put aside (Descartes, Newton) or taken back as just (transcendental) epistemic (intentional) forms or concepts (Kant).
} 
(ii) by reducing the final state of an evolutive process to its start-point state conditions (synchronic).

Both approaches are clearly based on different interpretations of the Closed World Thesis (CWT) that physical and/or formal systems are closed systems. ${ }^{16}$

Nowadays, there is wide agreement on the non-linear and dissipative with informationally open feature of fundamental physical processes (state-transitions systems) (LANDSMAN, 2017). The relevant point of the physics such processes is that it abandons the modern paradigm based on the view that natural processes are deterministic, in the sense discussed above. Indeed, the interplay between Quantum Field Theory (QFT) of dissipative systems - i.e., that operate far-fromthermodynamic equilibrium - and NLT is the core. Such a sort of systems are characterized by a) SBS mechanism and b) the formation of emergent structures, because of interacting quanta (gauge bosons) that show long-range correlations (unitary interactions) - think of EPR experiment (EINSTEIN et al., 1935; BOHM, 1951; Bell, 1964).

Del Giudice et al. (2009) argue that the most basic physical level, the quantum field, is itself constantly unstable (far-from-equilibrium) and that it constitutes the energetic openness condition for any physical system. Accordingly, the interplay between QFT and NLT lies on the Third Principle of Thermodynamics (TPT). The argument proceed as follows.

H. TPT is the conceptual basis of foundamental physics;

Then,

C1. It is impossible for any physical system to dynamically reach a state of no interactions with the environment or a state of no actuation of energy;

$\mathrm{C} 2$. The concept of inert isolated body is declined in fundamental physics.

C3. The notion of mechanic or absolute void results a mere abstraction:

Consequently the vacuum becomes a bridge that connects all objects among them. No isolated body can exist, and the fundamental physical actor is no longer the atom, but the field. (DEL GIUDICE et al., 2009, p. 1876).

Therefore, the existence of the isolated body, as a pillar of classical physics, results a mere abstraction from the modern bias of linearity operated by the constant application of the so called linearization methods ${ }^{17}$ to physical processes (LANDSMAN, 2017, p. 367).

\footnotetext{
${ }^{16}$ If we conceive mathematical theory as formal system, that is, a system based on primitive truths that are given once for all and cannot change, and whose development consists entirely in deducing conclusions from them, i.e., the whole of a mathematical theory is contained in its primitive truths, then, by Gödel's first incompleteness "mathematical theories cannot be closed systems" (CELLUCCI, 2013, p. 218).

${ }^{17}$ Linearization methods tend to favor the formulation of physical processes in terms of linear differential equations. For instance, the asymptotic condition by which the distance between any
} 
Clearly, this account contrasts with any modern and any reductionist approach to physics and, therefore, with CWT.

\section{EMERGENCE BY DUALITY}

The physics of dynamic non-isolated systems is based on the mechanism of symmetries (patterns) formation that supposes symmetry breakings (SBS) (LANDSMAN, 2017). SBS mechanism is about "local" symmetries (or reversible maps), and this leads to a local formation of stable patterns. It turned out that the notion of duality, as it is conceived formal sciences - in particular in CT - may play a relevant role here, as Landsman witnesses in the preface of his (2017, viii), speaking of quantum duality from the point of view of operator algebras.

In meta-algebraic contexts duality may be seen as a relation between two particular mathematical categories, one being that of algebra and the other being that of its dual, coalgebra. If algebra was discovered since ancient times and, later, applied to the physics of isolated systems (viz., Newtonian mechanics), another category of mathematical tool has been discovered within CT in very recent times ${ }^{18}$ : that of coalgebra. Coalgebra(s) is(are) dual to algebra(s).

To come back to the duality system-environment, such a duality seems to be well captured by mathematics through the duality between algebra and coalgebra by means of a link, a connection between the pairs system-environment and algebra-coalgebra that seems to be not just a matter of chance, of subjective choice. It may be expounded, roughly, as follows. On the one side, the physical system provides us information about the initial conditions of its process evolution, i.e., position and motion; these, in turn, provide us just information about maximal conditions of its evolution, namely the range of all the many states such system may reach at a fixed evolutive point (in future). ${ }^{19}$ On the other side, the physical environment provides the final conditions, namely the minimal conditions under which the system is made stable, by restricting step by step, phase by phase the range of the possible states defined by the system. By this way a new symmetry is reached. It is exactly thanks to the constant matching between initial and final evolutive conditions that the stability of the physical process(es), once reached, is maintained. By this, we say that it "emerges" an actually existing (physical) entity: a contingent object. In this context, as we will see, in order to give a formal representation and description of such dual behavior, CT provides us the existence of two very appropriate mathematical objects: the initial algebra and the final

two system tends toward infinite serves to ensure that any energetic (and informational, in classical physics) exchange between those systems tends toward zero, so to isolate them. Notice, Fourier's physical and mathematical researches were characterized by such methods. This, obviously, directly affects the very nature of such processes for which we look for the prediction of their evolution (i.e., final states).

${ }^{18}$ See (ABRAMSKY, 2012, p. 15). Think that Eilenberg \& Mac Lane (1945) introduced categories for the first time and in a purely auxiliary fashion, as preparation for what they called "functors" and natural transformations.

${ }^{19}$ Recall the system shows a non-linear behaviour, thus it has not a univocal state at a certain fixed evolutive point. 
coalgebra (of an endofuntor) that represent maximal and minimal objects (i.e., sets obtained by union and product) respectively. ${ }^{20}$ The fact that those objects are proved to exist by relative theorems means the mathematical representation of physical duality may be always captured by such formal duality. Further, by CT, the notion of emergence itself may be captured as far as final and initial objects of categories, or categories themselves, can be always related by a third category $^{21}$ that of indexing functors - so to ensure the mapping ${ }^{22}$ of all the elements and morphisms (relations) of one category into the other (possibly the same). Thus, by such a matching ${ }^{23}$ between the elements of the pair algebra-coalgebra, this third further category ensures the formal description of emergence and stability: a new object (i.e., a new category) emerges (to existences) by duality.

\subsection{THE CONVERGENCE OF ML AND CT}

The reason for focusing on the interplay between modality and duality is twofold: (1) ML is recognized to be the logic of coalgebras; ${ }^{24}$ (2) Coalgebra is something more than just the dual notion of algebra, since it generalizes algebra: given that any functor (a morphism of morphisms) can be employed in it (see ABRAMSKY, 2010 and VENEMA, 2007).

Coalgebra is, then, the common term between ML and CT. Contrary to algebra(s), coalgebra has a very interesting feature: it is finitary in essence. Indeed, a generic functor admits a final coalgebra if the coalgebraic category related to that functor has a final object ${ }^{25}$ (VENEMA, 2007), that is to say an end point. In dual terms, such a final object constitutes a term "of convergence" for the evolutive process of any non-isolated system.

But a further point of convergence between ML and CT may be found out. And it may be individuated by two issues: one about the meaning of mathematical existence in CT and another about the interpretation may be given to ML.

- About existence within CT: the axioms of CT are formulated purely in terms of the algebraic operations (arrows), without any reference to 'elements' of the objects (ABRAMSKY, 2010).

This makes CT very different from set theory, where existent objects (sets) have a primacy with respect to any relations (morphisms) that can be defined over them, for no assertion of such existence is made by CT: CT is not assertory, but

\footnotetext{
${ }^{20}$ A particular homomorphism, one from and to one and the same category.

${ }^{21}$ Notice, this is exactly what the quantum mechanics (QM) cannot do. In fact, QM does not have the formal resources to unify two Hilbert spaces, as it happens here by this third category. In this sense, QM and Quantum Field Theory (QFT) are not antagonist.

${ }^{22} \mathrm{Via}$ a diagonal functor.

${ }^{23}$ This matching has as its (natural) interpretation in QFT the doubling degree of freedom by which the degree of freedom (dimensions) of the Hilbert space that describes the system.

${ }^{24}$ In fact, the notion of unfolding that characterizes coalgebraic functors can be seen as an accessibility relation over Kripke models in case it is a bounded morphism, i.e., a bisimulation (see BLACKBURN and van BENTHEM, 2007; VENEMA, 2007).

${ }^{25}$ That is, a coalgebra, say $Z$, such that from every coalgebra, say $A$, in the category, there is a unique homomorphism from $A$ to $Z$.
} 
rather hypothetical, about mathematical existence. CT axioms are just "putative", and "not absolute (as ZF and the likes) assertions about its objects (the meaning of its terms)" (HELLMANN; 2003, p. 9-11). Thus, CT does not constitute a theory of mathematical existence and then modalisation and process interpretations are plausible as well as well accepted in such a formal framework. This latter point on modality and process interpretation may be further strengthened by the second issue.

- About the modal interpretation: ML is usually interpreted by Kripke models, i.e., the so called relational interpretation of ML. But, "the most influential ways of thinking about Kripke models" is "to give them a process interpretation", ${ }^{26}$ for the most important notion of ML, that of bisimulation, may be seen as "a natural notion of process equivalence" (BLACKBURN; van BENTHEM, 2002, p. 14). ${ }^{27}$

What, then, makes technically converge CT and ML is, definitely, the category of coalgebra, via bisimulation and duality. In fact, according to Venema (2007, p. 390):

\begin{abstract}
This set-up enables the canonical definition of two notions of equivalence between coalgebras, namely, bisimulation and behavioral equivalence. As we will see as well, the definitions make the concept of a coalgebra very similar to that of an algebra. However, if one makes this connection mathematically precise, it turns out that coalgebras over the base category $C$ are dual to algebras over the opposite category $C^{o p}$. [...] Given the nature of coalgebra as a very general model of state-based dynamics, there is a natural place for modal logic as a formalism for reasoning about behavior.
\end{abstract}

\title{
4 THE PARADIGM SHIFT: CT VS ZF AS FOUNDATIONAL THEORY
}

Sometimes scientists as well as philosophers confuse "formalism" with "mathematisation". What is rigorous and abstract, i.e., symbolic, is not necessarily formal as far formal systems are concerned only with syntactical issues, without any reference to meaning. Surely, it is not in this way that physicists work constructing representation of the world with permanent reference to meaning (physical interpretations). This is, physicists "propose mathematical structures, and not formal systems. Between the two there are at least the great theorems of incompleteness, which separate structural construction principles [viz., well-order of integers in Peano's arithmentic] from formal deductions." Thus, "Gödel's theorem of incompleteness breaks the alleged symmetry" hypothesis "between formal language and ontologising semantics" (BAILLY; LONGO, 2012, p. 32-33). This, I think, inspires the ontological investigation presented here which started from the

\footnotetext{
${ }^{26}$ Which means that we view models as collections of computational states, and the binary relations as computational actions that transform one state into another.

27 That makes two (modal) models (modally) identical.
} 
abandonment of the old axiomatic approach to foundations employed in many formal investigations of ontology. In this sense, CT may constitute a paradigm shift for formal ontology. CT - as the study of structures and their behaviour - embodies such a paradigm shift, for it "is not just another mathematical theory, but a way of mathematical thinking, and of doing mathematics". CT, indeed, embodies a different approach to formal sciences: "the shift from the set-theoretic to the categorical perspective provides the most dramatic example we possess" (ABRAMSKY, 2010, p. 88). Accordingly, CT is not to be thought as an alternative foundational scheme, but as "an alternative to foundational schemes in the traditional sense", where for "traditional" it is intended 'reductionist' or 'axiomatic'. Awodey (2004) is clear when he answers to criticisms by Hellman (2003) by claiming that his "proposal [...] is not to prefer this or that foundation, but to use CT to avoid the whole business of "foundations" (AWODEY, 2004, p.2). Awodey appeals to the reason that "'categorical foundations' is more bridge-building than foundation-building" (AWODEY, 2004, p.2). As if in building of a house, the basis looses any concrete meaning and operative role without assuming the act of building itself, according to an organisation of the building blocks and their functions. CT strategy is more like a bridging in that it specifies, for any given theory, just the relevant purpose oriented information without looking to the inner syntactic 'structure' of the objects involved.

To make things clearer and explicit, it may be useful to stress a little more that the great shift is from the axiomatic approach to the constructive (semantic) one. Cellucci (2013, pp. 11-13) argues that the limitations of the axiomatic (traditional) view of formal sciences based on set theory (ST) - due to Gödel's theorems - are the following:

1 ST does not permit to distinguish between mathematical theories in terms of their significance (syntax dependence);

2 ST leads to a fragmentation of the research;

3 ST cannot explain the success of the application of mathematics to physics.

It is not a matter of chance that Abramsky and Tzevelekos, in the introduction of their (2011), claim that CT, in turn, offers the following advantages:

1' CT gives a syntax-independent view of the fundamental structures of logic, and opens up new kinds of models and interpretations;

2' CT organises mathematics revealing new connections among structures and new structures (i.e., coalgebras);

3' CT offers new ways of formulating physical theories in a structural form.

As one can see, there is a one-to-one correspondence ST-limitations/CTadvantages. In order to discuss this issue, a line of argumentation may be based on what follows:

- The failure of any axiomatic attempt to represent mathematical objects as sets rests on two interrelated points:

(i) ST is too concrete. 
That is, ST involves irrelevant formal details linked to its syntax, i.e., it is just a particular coding - there are many others - thinks of the set theoretic definition of product: it depends on that of ordered couple, that, in turn, may be defined in many syntactically different ways with the consequence is that there is no one canonical definition of the former concept. Instead, on its side CT stresses 'how a math object performs' (always in the same way in any context where it exists), its behaviour and 'not what it is'.

(ii) ST, for (i), suffers a loss of generality

This is, it provides objects and properties dependent on definitions and axioms as they are syntactically expressed. On the contrary, in CT as a universal construction, if a math object exists in a given context (category), it has to be and it is unique up to unique isomorphism.

It is clear that CT goes beyond coding, for axioms are expressed in terms of the algebraic operations on arrows that is without any reference to existent 'elements' of the objects. What makes CT very useful and adequate for the formalization of dynamic non-linear systems is precisely its arrow-theoretic nature; its really being object independent: it may be seen as a (formalized) process theory (see HELLER, 2016). But, further CT possesses an interesting meta-mathematical characterization, as we will see in the next subsection.

\subsection{CT VS ZF: AN ANTI-PLATONISIIC FOUNDATION}

CT is even a suitable meta-language for non platonist ontologies. The realist foundation of formal sciences has been and is still displayed in many ways. About the set theoretic approach to logic, the most common is the second-order logical foundation of set theory (of mathematics) (see SHAPIRO, 1991; FERRARI, 2018, for a debate). A second, position may be that of Quine who, although refusing secondorder speech, considered first-order ST as a form of Platonism because sets are to be considered abstract (purely formal) entities (see COCCHIARELLA, 2001, p. 127). However, set-theoretic semantics has to engage with higher-order notions such as 'function of functions' or 'class of classes' for granting categoricity to the theory. ${ }^{28}$ This Platonist approach to foundations is due to an intrinsic feature of $\mathrm{ZF}$ - and the like - that ensures priority to set total ordering by some appropriate axioms, such as the regularity axiom ${ }^{29}$ or the axiom of choice. ${ }^{30}$ On the contrary, the category Set admits also non-standard sets of the sort of those that satisfy the anti-foundation

28 The power of categoricity is weakened by the semantic incompleteness of second-order logic (due to Gödel's theorems), i.e., two model for the same second-order theory are always isomorphic but the set of axioms (or rules) for second-order logic is not complete i.e., not fixed once for all; hence, the syntactic resources are not enough for proving all truths.

${ }^{29}$ Every non empty set A has an element B such that B and A are disjoint. By this axiom, in ZF it is avoided the case: (i) a set is member of itself; (ii) there exist an infinite descendent succession of sets. That is, it ensures the implicit existence of $U r$-elements. Regularity axiom is sometimes called 'foundation axiom'.

30 That is equivalent to a formulation of comparability, by means of the domination function, between sets. Recall that comparability, antisymmetry and transitivity make a relation a total/linear order. 
axiom (AFA), and called 'non-well founded' (NWF) (see ACZEL, 1988). In NWF-set account, set self-belongingness and unbounded chains of set(s) are possible - i.e. $\Omega={ }_{d f}\{\Omega\}$, thus, "unfolding" this equation would give $\Omega=\{\{\{\ldots\}\}\}$ (ACZEL, 1988, p. 6). Therefore, in NWF-sets only partial orderings are possible. Reflexivity of partial orderings makes ordering relation themselves 'objects' in the category. Thus, for reflexivity, notions as 'morphism of morphisms' in a NWF-set theoretic semantics are by no means per sé higher-(second-)order notions, contrary to (standard) set theoretic semantics. Accordingly, within this approach, the metalanguage of logical and mathematical theories does not imply per sé any quantification over second-order predicates and functions.

By this way a second sort of realism is possible, one very far from Platonism.

To gloss the theoretic power of NWF-sets and their nexus with ML via coalgebras, let me end this section with the following quotation from Blakburn et al (2002, p. 48):

Non-wellfounded sets and many other notions, such as automata and labeled transition systems, have been brought together under the umbrella of co-algebras [...] which form a natural and elegant way to model state-based dynamic systems. Since it was discovered that modal logic is as closely related to co-algebras as equational logic is to algebras, there has been a wealth of results reporting on this connection.

\section{ML AND COALGEBRA}

The duality algebra-coalgebra may serve to explain much of the interaction between ML and algebra. The coalgebraic perspective on ML, with respect to dynamic non-isolated systems is commented by Venema (2007, p. 332):

Coalgebras are simple but foundamental mathematical structures that capture the essence of dynamic or evolving systems. The theory of universal coalgebra seeks to provide a general framework for the study of notions related to (possibly infinite) behavior such as invariance, and observational indistinguishability. When it comes to modal logic, an important difference with the algebraic perspective is that coalgebras generalize rather than dualize the model theory of modal logic. Many familiar notions and constructions, such as bisimulations and bounded morphisms, have analogues in other fields, and find their natural place at the level of coalgebra. Perhaps even more important is the realization that one may generalize the concept of modal logic from Kripke frames to arbitrary coalgebras. In fact, the link between (these generalizations of) modal logic and coalgebra is so tight, that one may even claim that modal logic is the natural logic for coalgebras just like equational logic is that for algebra. 
What makes coalgebras so relevant simultaneusly for evolutive systems applications, the related ontology, and for the analysis of concepts such us that of emergence (by duality) comes from its very definition. In fact, whereas the algebraic operations adopted in the definition of algebras are ways to construct complex objects out of simple ones, coalgebraic operations, going out of the carrier set, should be seen as ways to unfold or observe objects. ${ }^{31}$ Such a point may be made clearer by thinking of the notions of mathematical induction and, its coalgebraic dual, coinduction ${ }^{32}$ and how they are respectively related to initial and terminal objects and to each other. Mathematical induction, indeed, is a way to construct objects up to infinity by means of the successor operation, i.e., the demonstration of the validity of the application of the induction step, $n+1$, to any previous one, $n$, given an initial object a base for induction. For example, given that some property holds for an initial object, in some sense, one may say that induction forces the property to hold even at the next step as when one says "There is no 2 without 3". Coinduction, differently, given a final object, select those steps built up by induction that provide the real construction of a finitary (the final object exists!) mathematical object. As when one notices that reality works differently from mathematics by asserting "No, there is no 3 without 2!". But, since the class of NWF-sets is the final coalgebra of the power-set functor, ${ }^{33}$ and NWF-sets defined as infinitely descending chains, just if one makes work induction and coinduction together, algebra and coalgebra together will obtain a finitary mathematical object and then a not necessarily actualist foundation of mathematical existence. By this way there is no need to assume the existence of all the infinite objects postulated by induction.

Unfortunately, not every functor (operation between categories) admits final coalgebras, i.e., Set-endofunctors involving the power-set functor and in particular, it is valid in ML contexts - there is no final Kripke frame or model. Nonetheless, it is a Fact that every small set functor ${ }^{34}$ admits a final coalgebra. ${ }^{35}$ The notion of smallness is easily seen to be equivalent to the instantiation in Set of the more general notion of accessibility - that characterizes Kripke relational semantics for ML.

\footnotetext{
31 This explains the central role of the notion of behaviour in the theory of coalgebras.

32 The principle of coinduction, dual to induction, serves both as an important proof tool and as an elegant means of providing definitions. As a definition principle, coinduction is based on the existence of a unique homomorphisms into the final coalgebra of an endofunctor.

33 A functor admits a final coalgebra if the coalgebraic category related to that functor has a final object, that is, a coalgebra $Z$ such that from every coalgebra $A$ in the category, there is a unique homomorphism from $A$ to $Z$.

34 "Examples of small functors abound; for instance, whenever we replace, in a Kripke polynomial functor, the power-set functor by a bounded variant such as the finite power-set functor, the result is a small functor. For instance, the finite power-set functor $\wp_{\omega}$ is $\omega$-small" (see VENEMA, 2007, p. 396-397).

35 "As one of the immediate corollaries of this Fact, the categories of image finite frames and image finite models, which can be represented as coalgebras for the functor $\wp_{\omega}$, and $\wp(\operatorname{Prop}) \times \wp_{\omega}$, respectively, have final objects" (see VENEMA, 2007, p. 396-397).
} 


\section{CONCLUSION}

Let me draw some conclusions. Recall that:

- CT generalizes set theory, being not object dependent;

This means that the employment of CT leads to a non-reductionistic way to provide a foundation in formal sciences, i.e., one that does not reduce a system/object into one another but that stresses the structural identities through morphisms. In particular, the notion of duality plays a very relevant role for what concerns analogies and generalization: the category of coalgebra of an endofunctor is, in fact, dually equivalent to the algebra of the controvariant endofunctor.

- NWF-sets generalize ZF-sets, and the like, with respect to CT (i.e., Set).

This happens for reflexivity and partiality has primacy to totality within the categorical analysis of sets. Partial functions and relations seem, thus, to be very foundative in this context.

- Coalgebra generalises algebra.

For any functor may be employed. This provides a link between CT and ML. The notions of unfolding, that characterizes coalgebraic functors, can be seen as an accessibility relation over Kripke models just in case it is a bounded morphism (bisimulation), such as the finite power-set functor.

The mainstream debate concerning philosophy of mathematics and the associated formal ontology is actually divided between two main positions: Platonism and Nominalism. This work was aimed to show that by the notion of duality and its characterisation within CT a third position might be available. A position that assimilates in a new synthesis and develops the following main stands of the formers:

1. Platonisms assumes that properties and relations (universals) have a form of independent reality, that second-order logic is the logic of foundation for second-order theories are categorical;

2. Nominalism assumes that the world is made by/of bare objects for firstorder logic is the only right logic for the technical results reached in such logic: completeness (semantic) and compactness.

Both stances bring some technical difficulties: a) Platonism is dealt with inifinitary foundations and b) nominalism is dealt with Löwenheim-Skolem theorem, namely equivocity with respect to formal reference to and characterisations of formal (mathematical) objects.

From the epistemological analysis of foundamental physics the notion of duality arises as necessary for the description of dynamic non-isolated systems. Thus, by the employment of CT it is possible to provide a formal characterization that take for good what Platonism and Nominalism take for bad. On the one hand, CT as a Platonist theory does not consider the existence of bare objects as primary; on the other hand, CT as a Nominalist theory does not involve second-order speech. 
Thus, the modal feature of existence together with the CT notion of duality between algebra and coalgebra might lead to a very specific ontology, a dual one, that is finitary and emergentist (contingentist) about the reference to individual entities. In this sense, this third stance may said to be Aristotelian.

\section{REFERÊNCIAS BIBLIOGRÁFICAS}

ACZEL, Peter. Non-wellfounded sets. Stanford: CLSI Lecture Notes, 1988.

ABRAMSKY, Samson. Logic and categories as tools for building theories. Journal of Indian Council of Philosophical Research, Issue on Logic and Philosophy Today, v. 27, n. 1, p. 277-304, 2010.

ABRAMSKY, Samson; TZEVELEKOS, Nikos. Introduction to categories and categorical logic. In: New Structures for Physics. COECKE, Bob. (ed.). Lecture Notes in Physics, v. 813. Dordrecht: Springer-Verlag, 2011. p. 3-94.

AWODEY, Steve. An answer to Hellman's question: "Does category theory provide a framework for mathematical structuralism?". Philosophia Mathematica, v. 12, n. 1, p. 54-64, 2004.

BAILLY, Francis; LONGO, Giuseppe. Mathematics and the Natural Sciences. London: Imperial College Press, 2013.

BELL, John S. On the Einstein-Podolsky-Rosen paradox. Physics, v. 1, n. 3, p. 195290, 1964.

BLACKBURN, Patrick; DE RIJKE, Maarten; VENEMA, Yde. Modal logic. Cambridge: Cambridge University Press, 2002.

BLACKBURN, Patrick; van BENTHEM, Johan. Modal logic: a semantic perspective. In: BLACKBURN, Patrick et al. Handbook of modal logic. Amsterdam: Elsevier, 2007. p. $1-84$.

BLACKBURN, Patrick., van BENTHEM, Johan, WOLTER, Frank (eds.). Handbook of modal logic. Amsterdam: Elsevier, 2007.

BOHM, David. The paradox of Einstein, Rosen and Podolsky. In: Quantum Theory. New York: Dover Publications, 1951. p. 611-623.

BRIDGMAN, Percy W. The logic of modern physics. New York: The Macmillan Company, 1958.

CELLUCCI, Carlo. Rethinking Logic. Dordrecht: Springer, 2013.

2014.

Knowledge, truth and plausibility. Axiomathes, v. 24, n. 4, p. 517-532,

COCCHIARELlA, Nino B. Logic and ontology. Axiomathes, v. 12, n. 1, p. 117-150, 2001.

Formal ontology and conceptual realism. Dordrecht: Springer, 2007. 
DEL GIUDICE, Emilio; PULSELLI, Riccardo M; TIEZZI, Enzo. Thermodynamics of irreversible processes and quantum field theory: an interplay for understanding of ecosystem dynamics. Ecological Modelling, v. 220, p. 1874-1879, 2009.

DUTILH-NOVAES, Catarina. The undergeneration of permutation invariance as a criterion of logicality. Erkenntnis, v. 79, n. 1, p. 81-97, 2014.

EILENBERG, Samuel; MacLANE, Sanders. General theory of natural equivalences. Transactions of the American Mathematical Society, v. 58, p. 231-294, 1945.

EINSTEIN, Albert; PODOLSKI, Boris; ROSEN, Nathan. Can quantum mechanical description of physical reality be considered complete? Physical Review, v. 41, p. 777-780, 1935.

ENDERTON, Herbert B. Elements of set theory. New York: Academic Press, 1977.

FERRARI, Francesco M. Some notes on Boolos' semantics: genesis, ontological quests and model-theoretic equivalence to standard semantics. Axiomathes, v. 28, p. 125-154, 2018.

FRAENKEL, Abraham A.; BAR-HILLEL, Yehoshua; LEVY, Azriel. Foundation of set theory. Amsterdam: Elsevier, 1973.

GARSON, James W. Quantification in modal logic. In: GABBAY, Dov M.; GUENTHNER, Franz. (eds.). Handbook of Philosophical Logic, v. 3. Dordrecht: Springer, 2001. p. 267-324.

GILL, Marie L. Aristotle's distinction between change and activity. In: SEIBT, Johanna (ed.). Process theories: crossdisciplinary studies in dynamic categories, Dordrecht: Springer, 2003. p. 3-22.

HALLETT, Micheal. Cantorian set theory and limitation of sizes. Oxford: Clarendon Press, 1984.

HELLER, Michael. Category free category theory and its philosophical implications. Logic and Logical Philosophy, v. 25, p. 447-459, 2016.

HELLMAN, Geoffrey. Does category theory provide a framework for mathematical structuralism? Philosophia Mathematica, v. 11, n. 2, p. 129-157, 2003.

HORGAN, John. Polymath Stephen Wolfram defends his computational theory of everything. Scientific American, March 5, 2017.

LANDSMAN, Klaas. Foundations of quantum theory. From classical concepts to operator algebras. Dordrecht: Springer, 2017.

LYRE, Holger. Holism and structuralism in U(1) gauge theory. Studies in History and Philosophy of Modern Physics, v. 35, n. 4, p. 597-624, 2004.

ROVELLI, Carlo. Reality is not what it seems: The journey to quantum gravity. New York: Penguin Random House, 2016.

NERST, Walther. The new heat theorem. New York: Dover Publications, 1969.

SEIBT, Johanna (ed). Process theories: crossdisciplinary studies in dynamic categories, Dordrecht: Springer, 2003. 
SHAPIRO, Stewart. Foundations without foundationalism: a case for second-order logic. Oxford: Oxford University Press, 1991.

VENEMA, Yde. Algebras and co-algebras. In: BLACKBURN et al. (eds.). Handbook of modal logic. Amsterdam: Elsevier, 2007. p. 331-426. 